\title{
Enhanced-DAB Converter: Comprehensive Design Evaluation
}

\author{
Armin Jafari \\ Power and Wide-band-gap \\ Electronics Research Laboratory \\ (POWERlab) \\ École polytechnique fédérale de \\ Lausanne (EPFL) \\ Lausanne, Switzerland \\ armin.jafari@epfl.ch
}

\author{
Mohammad Samizadeh Nikoo \\ Power and Wide-band-gap \\ Electronics Research Laboratory \\ (POWERlab) \\ École polytechnique fédérale de \\ Lausanne (EPFL) \\ Lausanne, Switzerland \\ mohammad.samizadeh@epfl.ch
}

\author{
Furkan Karakaya \\ Power and Wide-band-gap \\ Electronics Research Laboratory \\ (POWERlab) \\ École polytechnique fédérale de \\ Lausanne (EPFL) \\ Lausanne, Switzerland \\ furkan.karakaya@metu.edu.tr
}

\author{
Elison Matioli \\ Power and Wide-band-gap \\ Electronics Research Laboratory \\ (POWERlab) \\ École polytechnique fédérale de \\ Lausanne (EPFL) \\ Lausanne, Switzerland \\ elison.matioli@epfl.ch
}

\begin{abstract}
Soft switching in dual-active-bridge (DAB) converters enables their efficient operation at high frequencies, where the reduction in the size of magnetic components could result in ultra-high power densities. Nonetheless, losing softswitching at high frequencies results in severe efficiency degradations along with excessive thermal and electrical stresses on the transistors. In a previous work, we have presented an enhanced-DAB (E-DAB) topology along with an adjustable-tap high-frequency transformer to extend soft switching over wider voltage gains. The E-DAB achieved a peak efficiency of $97.4 \%$ without any complex modulation techniques, enabling a power density of $10 \mathrm{~kW} / \mathrm{l}\left(\right.$ or $164 \mathrm{~W} /$ inch $^{3}$ ) at $300 \mathrm{kHz}$. Here, we compare two different transformer geometries for their leakage inductance, quality factor and compatibility with the E-DAB. A theoretical gain-versus-power soft-switching characteristic for the E-DAB is verified by measurements of switching transients and amplitude spectrum analysis. The magnetic flux density and its distribution in the transformer core is analyzed using a finiteelement analysis (FEA) method and the on-load operation of electromagnetic tap changers is presented. E-DAB converters are of great importance to renewable energy harvesting, Li-ion battery chargers, future de distribution systems and smart grids due to their high efficiency, high power density and superior controllability.
\end{abstract}

Keywords-DAB, matrix transformer, tap changer, enhanced$D A B$, step up, GaN, quality factor, soft switching, wide gain range, planar transformer, electromagnetic relays

\section{INTRODUCTION}

Dual-active-bridge (DAB) dc-dc converters are capable of power regulation under different load conditions and voltage gains. Similar to an ac transformer, bidirectional-powertransfer capability and galvanic isolation make DABs promising for a wide range of applications including future dc distribution systems, flexible microgrids, electric vehicle (EV) bus converters and chargers, avionics, uninterruptable power supplies, renewable energy harvesting and telecommunications [1], [2]. High-performance Gallium-Nitride $(\mathrm{GaN})$ transistors offer low ON-resistance $\left(R_{\mathrm{DS}(\mathrm{ON})}\right)$, input-capacitance $\left(C_{\mathrm{ISS}}\right)$ and output-capacitance $\left(C_{\mathrm{Oss}}\right)$ losses, enabling a highly efficient soft-switching operation at high frequencies [3], [4]. The subsequent reduced size of magnetic components directly results in higher power densities [5]. However, operating a conventional DAB at high frequencies - and out of its softswitching regime - leads to excessive switching losses and risk of device failure. In other words, a conventional highfrequency DAB cannot operate efficiently over wide voltage gains.

Here, the design and performance of a previously proposed enhanced-DAB (E-DAB) [6] converter is assessed in greater details. The converter preserves its high efficiency over wider voltage gains and power levels by using an adjustable-tap highfrequency transformer [6]. The GaN-based E-DAB boosts the global efficiency without requiring complex modulation techniques [7], or addition of extra components which add to losses and complicate the high-frequency operation [8], [9]. The enhanced converter reached a peak efficiency of $97.4 \%$ together with a better efficiency compared to a conventional $\mathrm{DAB}$, for up to 2.8 times higher voltage gains. Moreover, the E-DAB increased the power-transfer capability of the converter by introducing an extra control parameter: the turns ratio of the transformer, which is instrumental for power regulation especially when the initial control parameter (phase shift) reaches its limit.

In Section II, we theoretically show the advantage of using an adjustable-tap transformer to extend the power-transfer capability and voltage gains under which an E-DAB maintains zero-voltage switching (ZVS). Section III presents two different high-frequency transformer geometries: a planar design and a matrix transformer realized by using Litz wires. We compare both designs for quality $(Q)$ factor, leakage inductance $\left(L_{\mathrm{r}}\right)$ and the variation of $L_{\mathrm{r}}$ with the taps. Section IV focuses on the design of compact $\mathrm{GaN}$ full bridges, including the printed-circuit-board (PCB) layout for minimized gate and power loops, together with the choice of driving circuit. In Section V, the theoretical prediction is verified by experiments, which highlight the importance of a gain versus power-transfer characteristic plane for design and control of E-DABs. Section VI discusses the application of EDAB for high-efficiency lithium-ion (Li-ion) battery chargers. Moreover, finite-element analyses (FEA) are conducted for the magnetic flux density and its distribution in the ferrite cores under various load conditions, and the on-load operation 
(a)
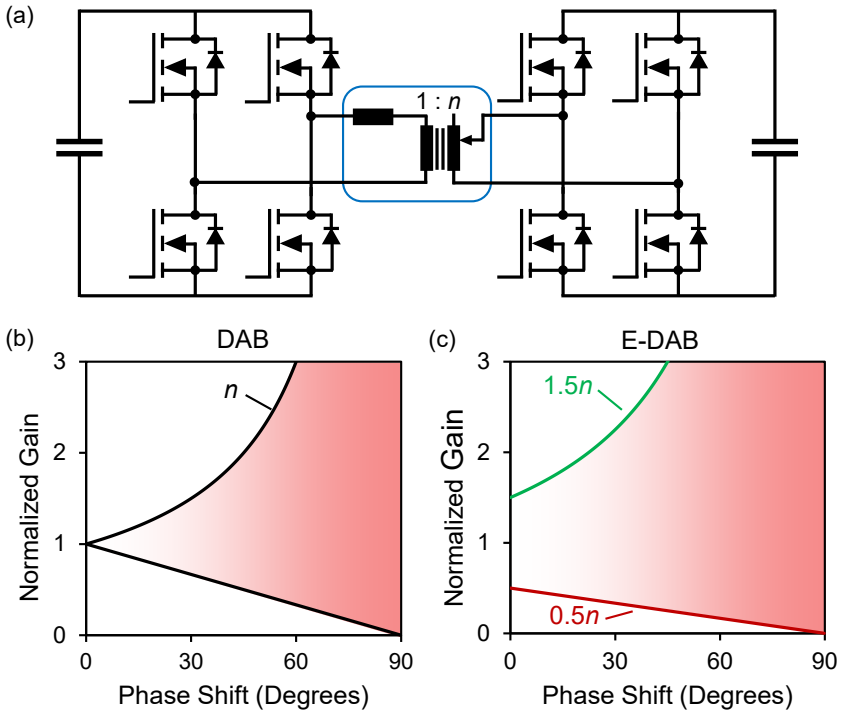

Fig. 1 Methodology for the operation of E-DAB converter presented in [6]. (a) The converter takes advantage of the transformer leakage inductance and employs a tap changer to increase the power-transfer capability and extend the voltage gains under which the converter is soft switched. Normalized gain versus phase shift is shown for (b) a convetional DAB with a fixed turns ratio $n$ and (c) an E-DAB with turns ratios adjustable by $\pm 50 \%$ from $n$. The shaded areas designate ZVS operation. The range is significantly extended in the case of using an E-DAB, especially at light loads.

of the electromagnetic (EM) relays (employed by the tapchanger mechanism) is demonstrated. Section VII concludes the most important aspects of the design and performance of the E-DAB converter. This work paves the way for designing high-performance high-frequency E-DABs.

\section{Methodology}

Power transfer in DAB converters is inductive and the phase shift between the voltages of the transformer on the primary and secondary sides, $\varphi$, determines the power as

$$
P=\frac{V_{\mathrm{IN}} V_{\mathrm{OUT}}\left(\frac{\varphi}{\pi}\right)\left(1-\frac{\varphi}{\pi}\right)}{2 n f_{\mathrm{SW}} L_{\mathrm{r}}}
$$

in which $V_{\mathrm{IN}}$ and $V_{\mathrm{OUT}}$ are primary and secondary dc-link voltages, respectively, $n$ is the transformer turns ratio, $f_{\mathrm{SW}}$ is the switching frequency and $L_{\mathrm{r}}$ is the inductance in series with the transformer (which could also be its leakage inductance). An E-DAB converter (Fig. 1a) uses the turns ratio of the transformer, $n$, as a secondary control parameter. Assuming the leakage inductance of the transformer does not vary with the turns ratio, the voltage-gain $(G)$ range under which the converter achieves soft switching can be defined as

$$
n\left(1-\frac{2 \varphi}{\pi}\right)<G<n /\left(1-\frac{2 \varphi}{\pi}\right)
$$

As (2) suggests, tuning the $n$ value extends the softswitching limits. Figs. 1b, c compare a conventional DAB with turns ratio $n$, with an E-DAB that has the capability to adjust its tap value by $\pm 50 \%$ from $n$. The shaded region represents the range in which the converters are soft switched. Incorporating a tap changer improves the efficiency in E-DAB, especially for light loads, as shown in Fig. 1c. Based on (1), the power is inversely proportional to the $n$. Therefore, when the phase shift reaches its limit and transferring more power is not possible with a conventional $\mathrm{DAB}$, the E-DAB still can transfer more power by adjusting $n$. A proper design of a tapped highfrequency transformer is crucial to the performance of the EDAB.

\section{HIGH-FREQUENCY TAPPED TRANSFORMERS}

The high-frequency transformer provides the voltage stepup/-down and galvanic isolation, and its leakage inductance if adequate - enables soft switching. For evaluating the theory presented in Section II, we designed tapped transformers with two different geometries; a planar design with PCB traces and a matrix transformer with Litz wires, as presented in Figs. 2a, $b$, respectively.

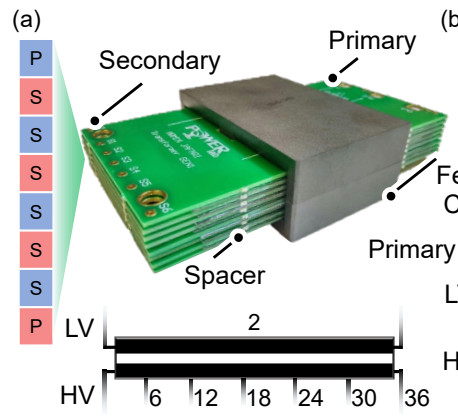

(b) Secondary Electromagnetic
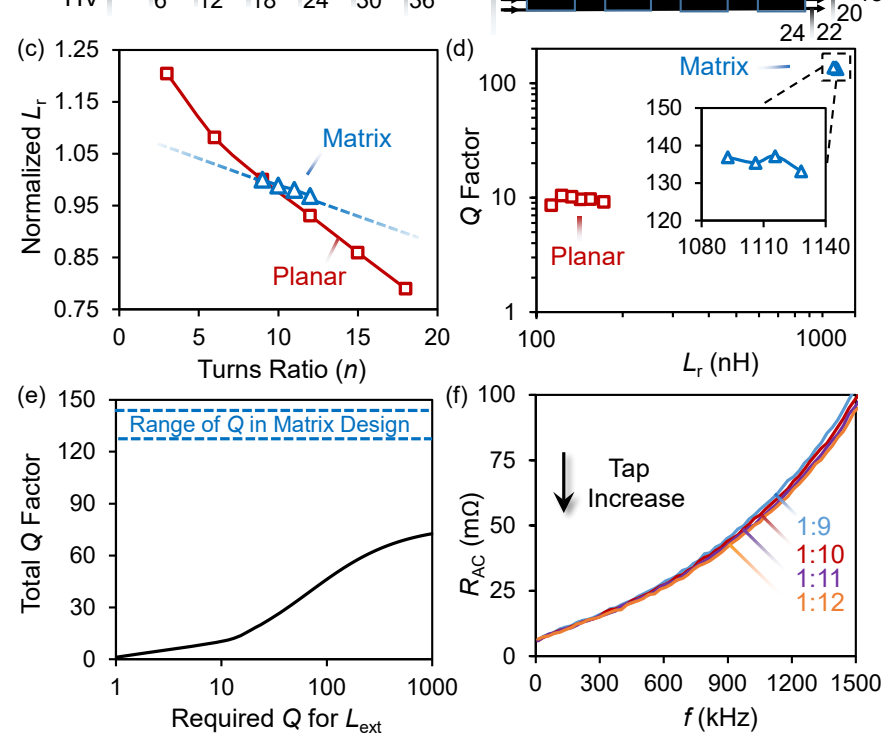

Fig. 2 Evaluation of different tapped transformers. (a) Planar transformer with PCB-trace windings and its arrangement. (b) Matrix transformer with Litz windings and its layout. (c) Normalized $L_{\mathrm{r}}$ versus $n$. The matrix design exhibits 2.6 times less variations compared to the planar design. (d) Measured $Q$ factor at $300 \mathrm{kHz}$. Both $Q$ factor and $L_{\mathrm{r}}$ are an order-of-magnitude higher in the matrix transformer. (e) Total $Q$ factor for $L_{\mathrm{r}}$ in the planar transformer in series with $L_{\text {ext }}$, versus the $Q$ factor required for the $L_{\text {ext }}$. Even adding $L_{\text {ext }}$ with $Q>1000$ does not increase the total $Q$ factor significantly. (f) $R_{\mathrm{AC}}$ over frequency for different taps in the matrix transformer. Using different taps does not affect $R_{\mathrm{AC}}$ over the presented frequency range, indicating that the use of different taps does not incur any extra losses in the E-DAB converter. 
The planar transformer consisted of E58/11/38 cores with 3C95 ferrite material, two low-voltage (LV) primary layers and six high-voltage (HV) secondary layers, as illustrated in Fig. 2 a. The ratio $n$ varied between 3 to 18 , with steps of 3 . The matrix transformer consisted of four toroidal cores $(\mathrm{R} 22.1 \times 13.7 \times 7.9)$ with $\mathrm{N} 49$ ferrite material. We chose Litz wires S1050/AWG42 for the primary windings and S66/AWG42 for its secondary. The ratio $n$ varied between 9 to 12, with steps of one. Fig. 2b presents the matrix transformer together with the EM relays. The tap changer is placed at the HV side of the transformer, in order to reduce the current stress on the EM relays. The bottom sections in Figs. $2 \mathrm{a}$, b present the winding arrangements.

We measured the leakage inductance for each design by a short-circuit test using Keysight E4990A impedance analyzer and 16047E test fixture. As Fig. 2c shows, the variation of $L_{\mathrm{r}}$ with $n$ is lower for the matrix design (by a factor of 2.6 times), which better complies with the assumption used in (2) regarding the invariant leakage inductance over different taps. $L_{\mathrm{r}}$ in Fig. 2c was normalized by its value at $n=9$ for both transformers. Furthermore, the leakage inductance value was about 10 times larger in the matrix design, as shown for different tap values in Fig. 2 d. $L_{\mathrm{r}}$ in the matrix design obtained one order-of-magnitude higher $Q$ factor at the target switching frequency (i.e. $300 \mathrm{kHz}$ ), which results in significantly lower losses (see Fig. 2d). To reach the same inductance as that of the matrix transformer, one needs to add an external inductor $\left(L_{\mathrm{ext}}\right)$ in series with the planar transformer. As Fig. 2e shows, even adding an extremely high-quality inductor $(Q$ factor $\sim 1000)-$ which could be highly challenging to design [10] - does not offer the same performance as the $L_{\mathrm{r}}$ in the matrix transformer. Thus, we chose the matrix transformer for the E-DAB design.

The winding ac resistance $\left(R_{\mathrm{AC}}\right)$ for the matrix transformer is shown in Fig. $2 \mathrm{f}$ for different taps. $R_{\mathrm{AC}}$ is low at the target switching frequency (i.e. $300 \mathrm{kHz}$ ) and its value does not vary much for different tap values, indicating that the variable-tap transformer does not incur any extra losses to the E-DAB.

\section{CONVERTER DESIGN}

To enable a high-frequency operation, we employed highperformance $\mathrm{GaN}$ transistors EPC2031 (60 V, $48 \mathrm{~A})$ and GS66502B $(650 \mathrm{~V}, 7.5 \mathrm{~A})$ for primary and secondary full bridges, respectively (Figs. 3a, b). The selection criteria for these switches is discussed in [11]. The gate drivers (SI8271) provided isolation between the power and control stages with high transient $(\mathrm{dv} / \mathrm{dt})$ immunities. The PWM signals were generated using a TMS320F28379D controller and were transmitted to the gate drivers by shielded MMCX cables and connectors [6]. To supply the gate drivers, we chose compact isolated dc-dc converters (ADUM5000) capable of withstanding up to $2.5 \mathrm{kV}$. They were mounted on the bottom layer and provided a 5-V supply for driving of the GaN transistors. Fig. $3 \mathrm{c}$ presents the optimized E-DAB converter with an overall size of $80 \times 50 \times 25 \mathrm{~mm}^{3}$. The tap-changer mechanism consists of four EM relays, each capable of switching about $2 \mathrm{~A}$. They were rated for more than $60 \mathrm{VA}$ of apparent power, appropriate for operation at the secondary-side of the E-DAB. To minimize the gate-loop and power-loop inductances, we designed fourlayer PCBs as illustrated in Fig. $3 \mathrm{~d}$ for the primary-side full bridge. To the same end, we used multi-layer ceramic

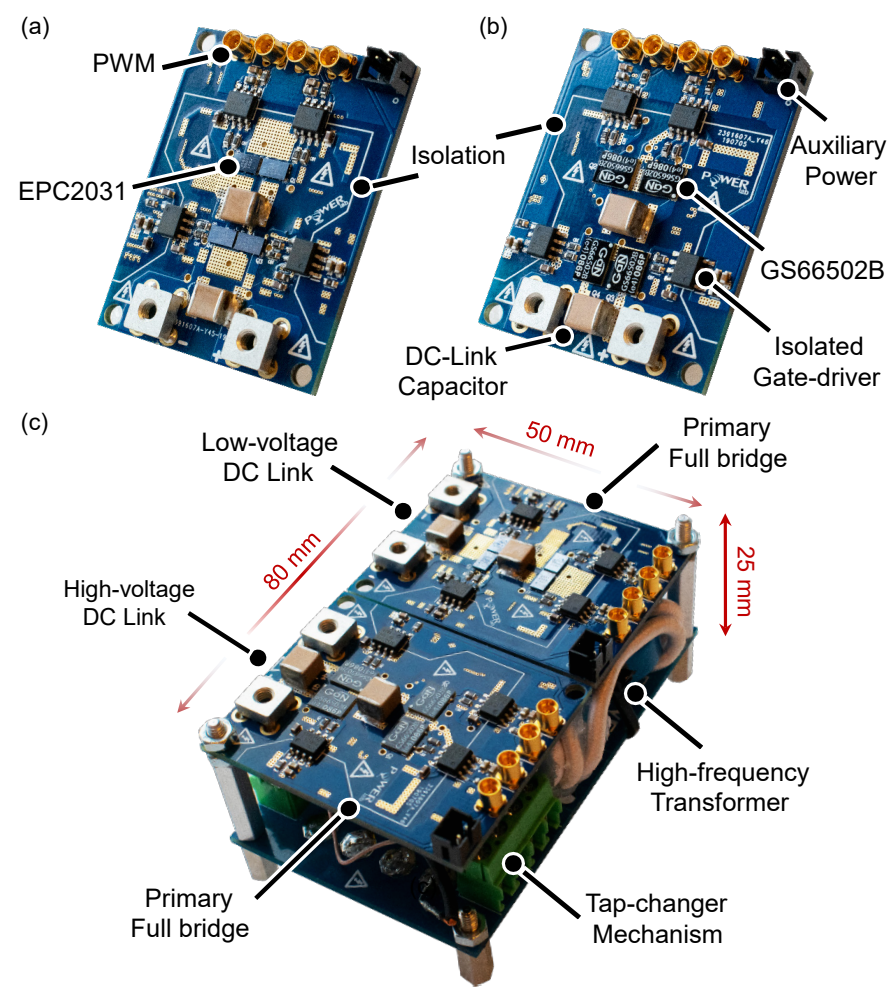

(d)

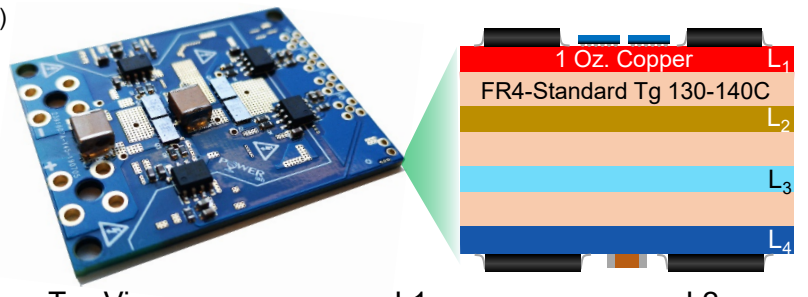

Top View

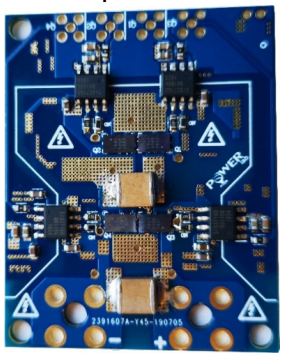

L3

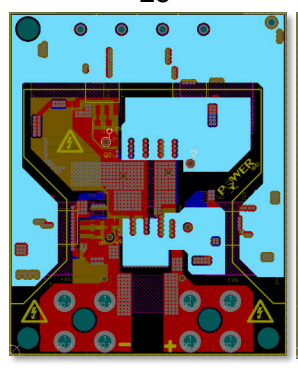

L1

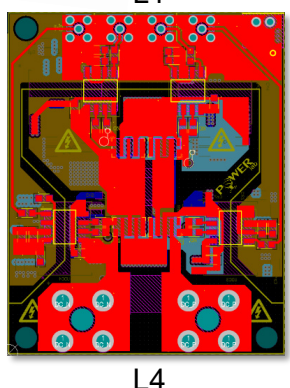

L2

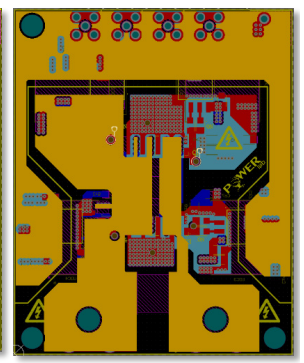

Bottom View

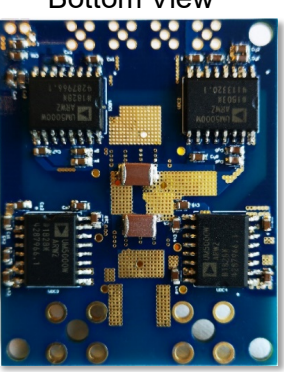

Fig. 3 The E-DAB converter consists of GaN transistors, a highfrequency tapped transformer and its tap-changer mechanism. (a) Primary full bridge at LV side with EPC2031 transistors. (b) Secondary full bridge at HV side employs GS66502B transistors. (c) The optimized E-DAB converter with a compact design. (d) Demonstration of the four-layer PCB layout. At high frequencies, it is crucial to minimized gate-loop and power-loop inductances. Together with a very high density, a fully-isolated PCB design was realized. Moreover, the components such as gate drivers can withstand up to $2.5 \mathrm{kV}$. 


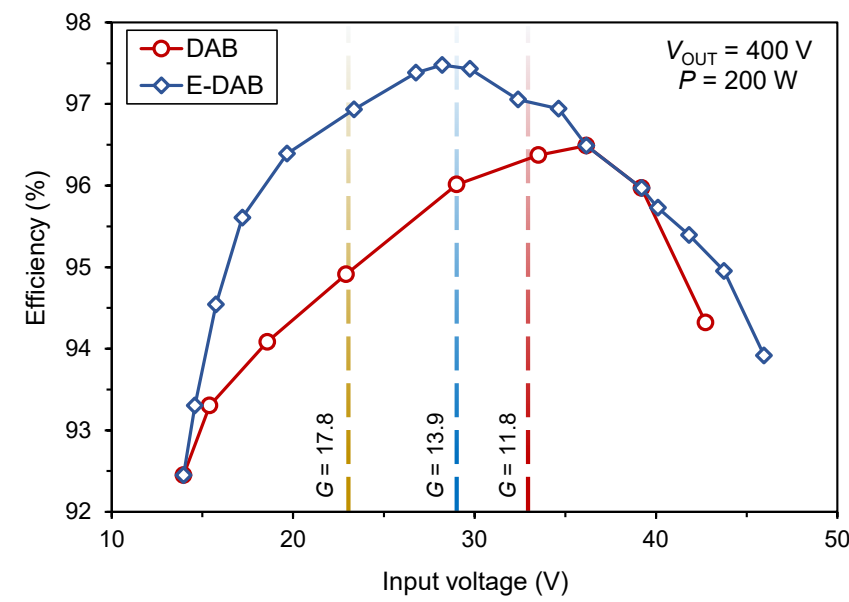

Fig. 4 Efficiency comparison of the conventional DAB and the E-DAB at the same light load of $200 \mathrm{~W}$ and HV dc-link voltage of $400 \mathrm{~V}$. The E-DAB outperforms the $\mathrm{DAB}$ over the entire input voltage sweep, corresponding to 2.8 times higher voltage gains compared the nominal 10x step-up in the DAB.

capacitors very close to the dc links and gate-driver supplies.
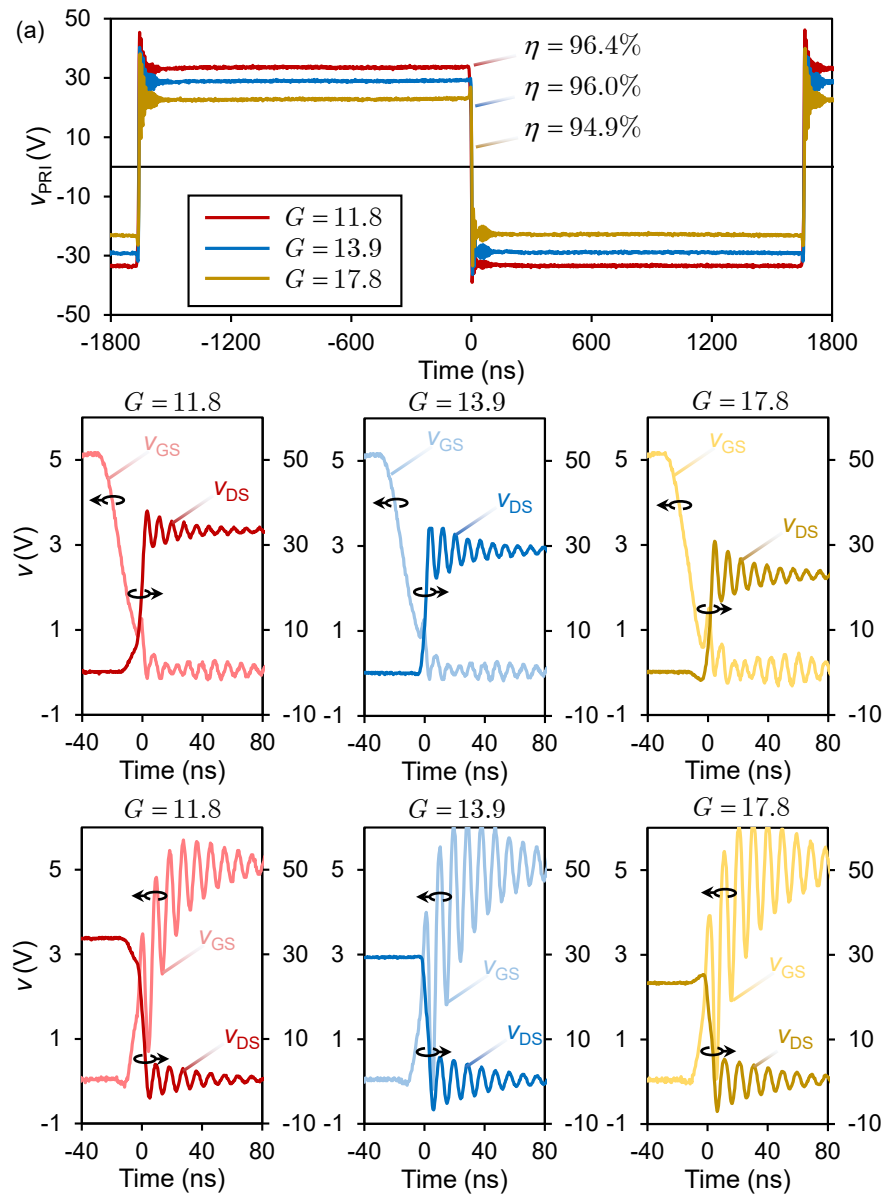

Fig. 5 Primary-side voltage waveforms of the DAB at $200 \mathrm{~W}$ for $G=$ 11.8, 13.9 and 17.8. (a) Transformer $v_{\text {PRI }}$ with the corresponding efficiencies. The $v_{\mathrm{GS}}$ and $v_{\mathrm{DS}}$ waveforms are shown for (b) turn-OFF and (c) turn-ON transients of two low-side primary switches. By increasing $G$, the partial ZVS behavior for turn-ON transient changes into hard switching, together with reverse conduction of the high-side device, leading to higher switching losses.
A similar high-density design was realized for the secondaryside full bridge. The copper thickness for each of the inner and outer layers was $1 \mathrm{oz}$ (or $\sim 35 \mu \mathrm{m})$.

In order to make an experimental comparison between the performance and efficiency of the E-DAB and the DAB without the risk of device thermal runaway, we used forced-air cooling at various conditions, especially those at the limit of transition from ZVS to hard switching, whereby the transistors were exposed to high levels of switching losses.

\section{EXPERIMENTAL VERIFICATION}

The converter was designed to operate at $300 \mathrm{kHz}$. We employed a single-phase-shift (SPS) modulation, whose simplicity is favorable for high-frequency operation. To verify the extension of soft switching to higher voltage gains as predicted by (2), we supplied a relatively light load of $200 \mathrm{~W}$ at an output voltage of $400 \mathrm{~V}$ with the E-DAB and a conventional $\mathrm{DAB}$. The conventional DAB was realized by using tap $n=10$ of the E-DAB during all the experiments.

Fig. 4 compares the efficiency of the E-DAB and the DAB
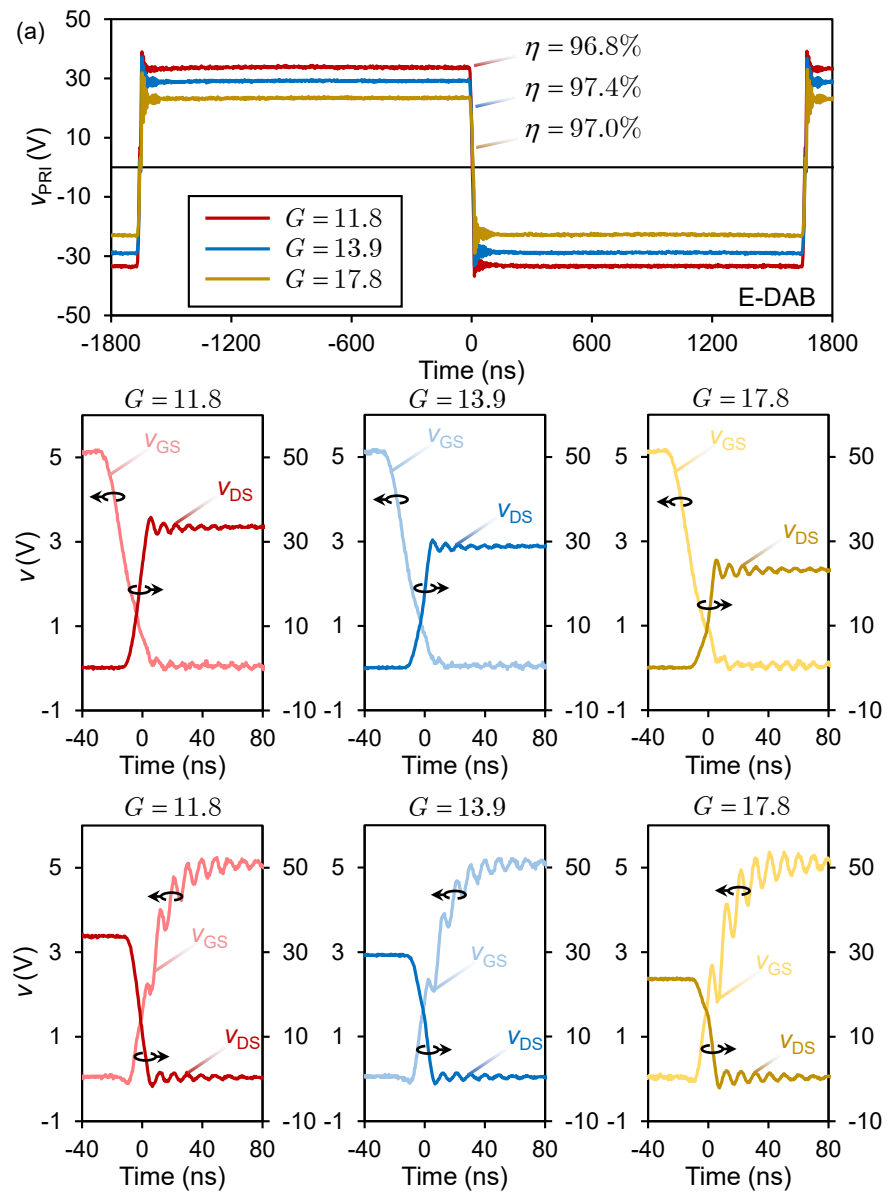

Fig. 6 Primary-side voltage waveforms of the E-DAB at $200 \mathrm{~W}$ for $G=$ 11.8, 13.9 and 17.8. (a) Transformer $v_{\mathrm{PRI}}$ with the corresponding efficiencies. The $v_{\mathrm{GS}}$ and $v_{\mathrm{DS}}$ waveforms are shown for (b) turn-OFF and (c) turn-ON transients of two low-side primary switches. By increasing $G$, the full ZVS behavior for turn-ON transient changes into a partial ZVS, which leads to higher efficiencies compared to the DAB at the same operating conditions. 

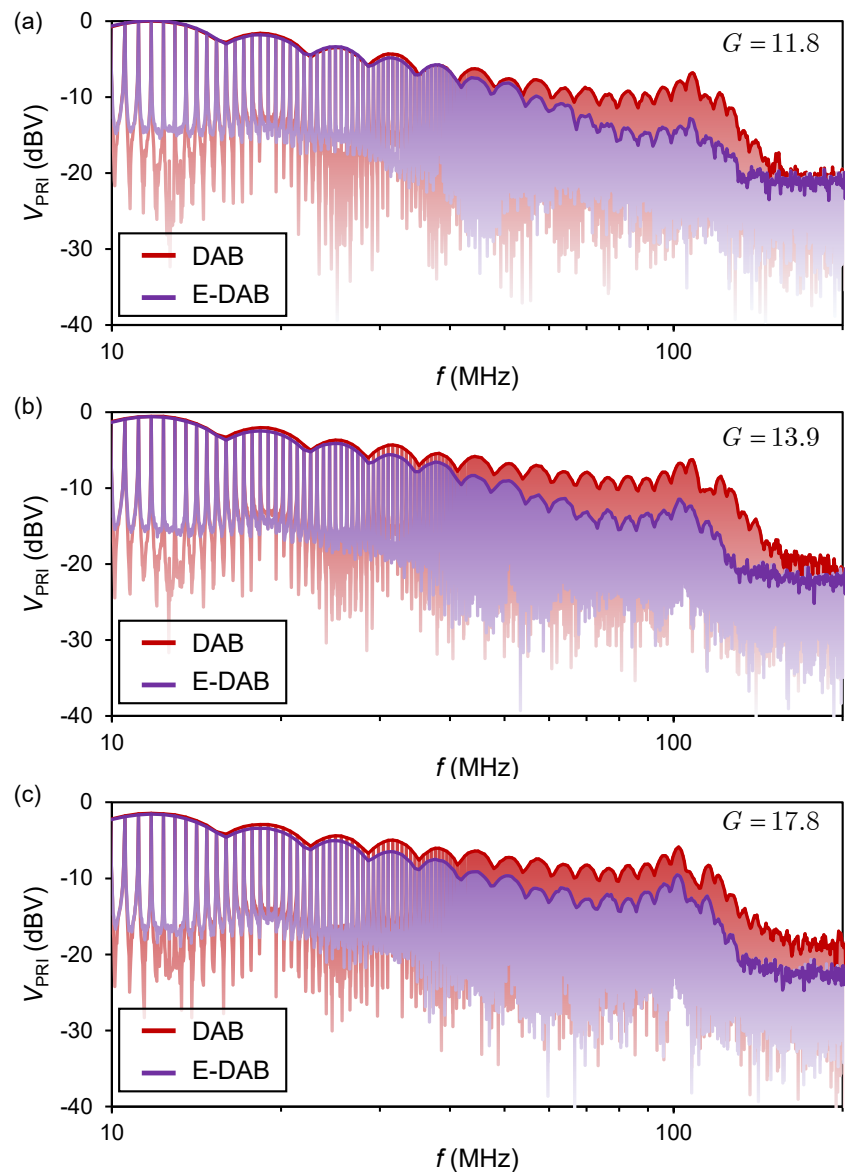

Fig. 7 Single-sided amplitude spectrum of $v_{\mathrm{PRI}}(t)$ from $10 \mathrm{MHz}$ to 200 $\mathrm{MHz}$, compared for the DAB and the E-DAB for $G$ values of (a) 11.8 , (b) 13.9 and (c) 17.8, corresponding to the waveforms presented in Figs. 5, 6. Extended ZVS in the E-DAB topology results in significantly lower amplitudes of high-frequency harmonics and thus higher overall efficiencies.

converters, where the E-DAB outperforms the $\mathrm{DAB}$ for the entire input voltage range, the equivalent of up to 2.8 times higher voltage gains compared to the conventional DAB. In addition, the peak efficiency was increased from $96.5 \%$ to $97.4 \%$. The subsequent reduction in the losses significantly reduces the risk of thermal runaway and device failure, particularly at light-load conditions and wide-gain operations.

Figs. 5, 6 compare voltage waveforms of the transformer and low-side transistors on the primary full bridge for three different gain values, corresponding to the dashed lines in Fig. 4. Primary-side transformer voltage $\left(v_{\mathrm{PRI}}\right)$ for DAB and E-DAB converters are shown in Figs. 5a, 6a as both were operated at $200 \mathrm{~W}$.

To observe the switching behavior, we have measured gateto-source $\left(v_{\mathrm{GS}}\right)$ and drain-to-source $\left(v_{\mathrm{DS}}\right)$ voltages of two lowside transistors using Tektronix TPP1000 1-GHz voltage probes, for turn-OFF (Figs. 5b, 6b) and turn-ON (Figs. 5c, 6c) transients. Higher gains result in lower efficiencies in the DAB, whereas the E-DAB achieves higher efficiencies as predicted by (2). Figs. 7a-c show the frequency contents of $v_{\text {PRI }}$ for both converters at $G=11.8,13.9$ and 17.8 , respectively.
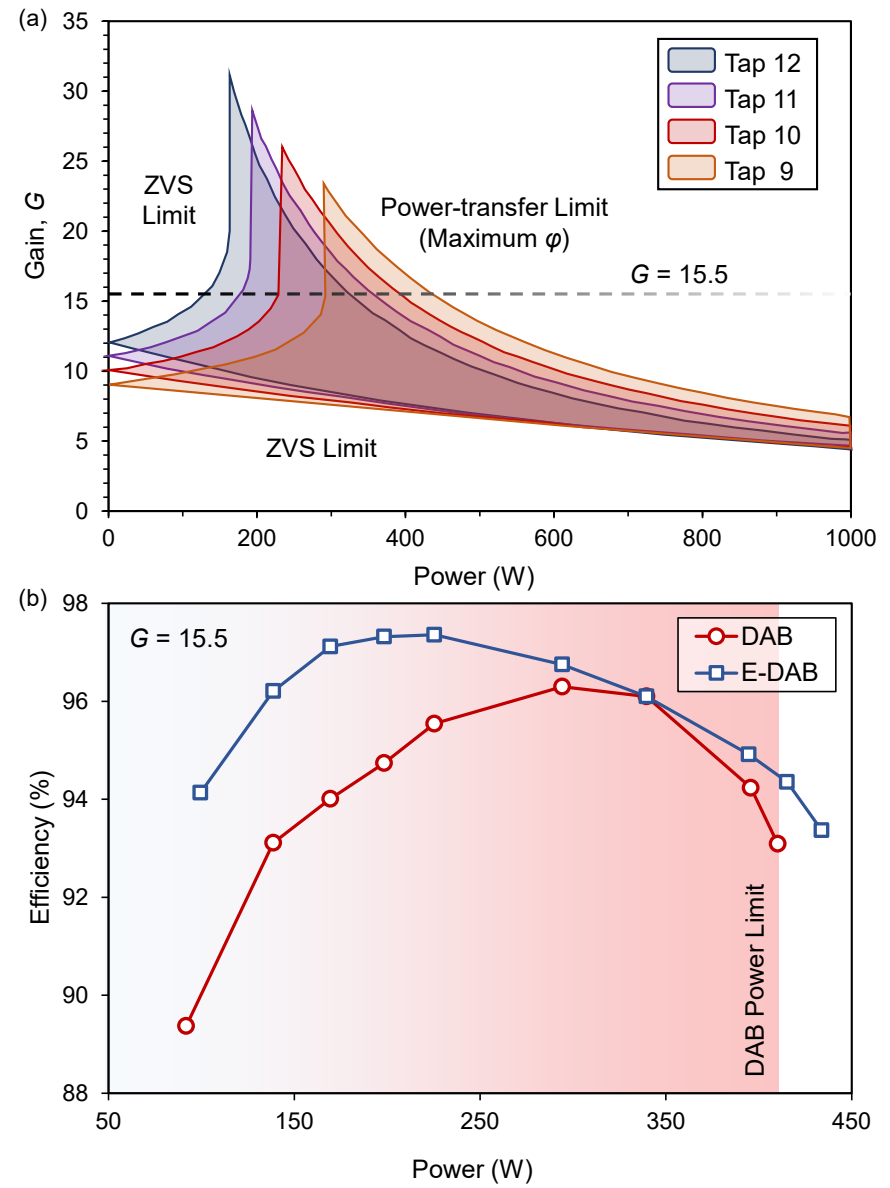

Fig. 8 Analysis of efficiency over different voltage gains and power levels [6]. (a) Gain versus power-transfer characteristics of the E-DAB converter. The shaded areas indicate ZVS operation of the E-DAB. (b) Experimental evaluation of efficiency versus power at a fixed $400-\mathrm{V}$ output voltage and $G=15.5$. The shaded area indicates the power-transfer limit of the DAB. Experimental results verify the prediction of gain versus powertransfer characteristics plane, as the E-DAB always achieved a higher efficiency.

Hard switching in the conventional DAB at higher gain values not only results in lower efficiencies, but also generates harmonics at frequencies between $10 \mathrm{MHz}$ to $200 \mathrm{MHz}$, which could intensify electromagnetic interference (EMI) issues in the converter. For instance, the $v_{\mathrm{GS}}$ waveforms of the $\mathrm{DAB}$ in Fig. 5c suffer from relatively larger oscillations compared to those for the E-DAB, shown in Fig. 6c. Such EMI issues could lead to false triggering and device failure, especially in GaN devices with low threshold and gate-breakdown voltages.

For a fixed output voltage, one can solve the limits for $G$ as indicated by (2) for different values of $\varphi$ and use (1) to obtain a gain versus power-transfer characteristic plane for the EDAB. Combined with the power-transfer limit due to the maximum $\varphi$, such a characteristic specifies the operating range in which the E-DAB is soft switched for its different $n$ values. The characteristic plane in Fig. 8a is presented for the E-DAB operating at a $400-\mathrm{V}$ fixed output voltage [6]. We experimentally verified the analysis by comparing the E-DAB with the DAB at a fixed voltage gain of 15.5. As compared in Fig. 8 b, the E-DAB preserved higher efficiencies compared to 
the $\mathrm{DAB}$ over the entire power sweep range and increased the efficiency by $5 \%$ at an extremely light load of $80 \mathrm{~W}$. Moreover, when $\varphi$ reached its maximum value in the DAB (i.e. 90 degrees), E-DAB could still transfer more power. The DAB power-transfer limit is indicated by the shaded area in Fig. 8b.

The gain versus power-transfer characteristic is an important analysis tool for the sizing, design, and control of EDAB converters. The tap values for the transformers can be selected based on gain and power requirements of the target application. Besides, a controller can maximize the efficiency of the E-DAB by selecting the proper values for $\varphi$ and $n$ at any given operating condition.

\section{DISCUSSION}

Here, we demonstrate the superiority of the E-DAB topology over a conventional DAB in high-efficiency charging of Li-ion batteries based on their specific charging profiles. Next, we address important operational aspects of the E-DAB converter, including the variation of transformer core losses at different load conditions and the dynamics of the tap changer.

\section{A. Application of E-DAB in ultra-efficient Li-Ion Battery Charging}

Li-ion batteries are rechargeable and commonly used in portable electronics, EVs and aerospace applications. A Li-ion battery is charged in two stages: constant current (CC) and constant voltage $(\mathrm{CV})$. During the $\mathrm{CC}$ phase, the charger applies a constant current to the battery at a steadily increasing voltage. Next, in the CV phase, the voltage is kept constant and the current gradually declines to zero, until a certain threshold for the current is reached.

Fig. 9 illustrates the gain versus power-transfer characteristic of the E-DAB with the two charging phases (i.e. $\mathrm{CC}$ and $\mathrm{CV}$ ). In this case, the E-DAB can charge a Li-ion battery in step-down mode, when the power is supplied from the HV side at $400 \mathrm{~V}$ to the $\mathrm{LV}$ Li-ion batteries (the charging profile of a single-cell Li-ion battery is scaled up in both voltage and current).

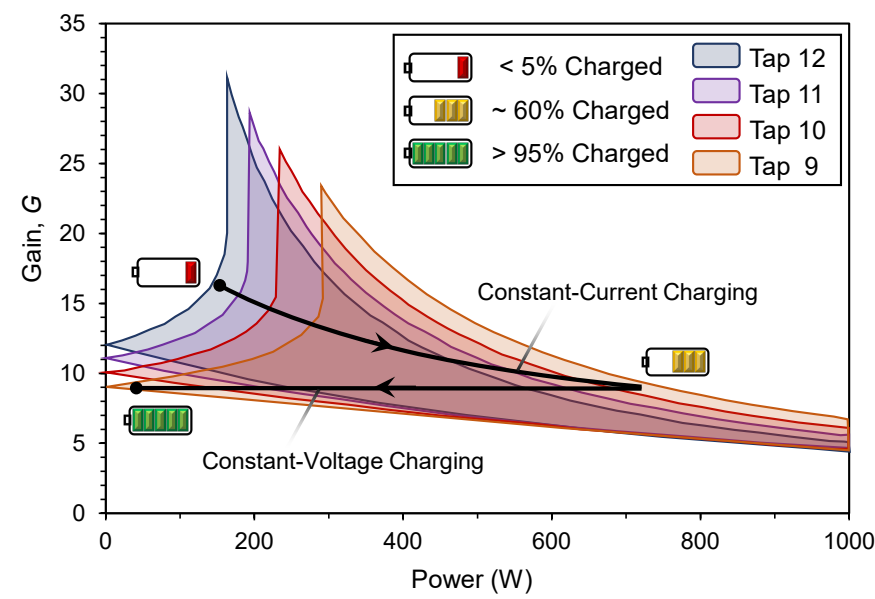

Fig. 9 Application of the E-DAB converter in ultra-efficient Li-Ion battery charging. By tuning the tap values, E-DAB can maintain soft switching over the entire $\mathrm{CC}$ and $\mathrm{CV}$ charging phases.
In $\mathrm{CC}$ phase, the battery is being charged and its voltage is increasing. Thus, the gain is reducing while the power increases. The E-DAB can achieve ZVS by shifting the taps to lower values and maintains a high efficiency over the entire charging process.

\section{B. Transformer core losses at light-load conditions}

It was shown in Fig. 8, that at a fixed voltage gain, E-DAB achieved higher efficiencies over the entire power range. We related this superior performance to the extended softswitching range for the E-DAB. It is worth investigating the transformer core losses, especially at light-load conditions, as a potential limiting factor for the efficiency of the E-DAB.

We used FEA simulations to obtain the distribution and amplitude of magnetic flux density $(B)$ inside the matrix transformer cores. Higher $B$ values result in increased core losses. Fig. 10 presents the FEA results when the matrix transformer was subjected to a $40-\mathrm{V} 300-\mathrm{kHz}$ sine voltage excitation at its primary side and different load resistances $\left(R_{\mathrm{L}}\right)$ were placed at its secondary side. After a certain reduction in the load, the transformer core operates similar to a no-load condition (with highest core loss, as there is almost no flux cancellation occurring in the core) with negligible increase of $B$ between $20 \%, 10 \%$ and $5 \%$ of full load.

Despite the absence of flux cancellation at light loads, the proper sizing of the transformer cores enabled achieving low levels of core losses, which could otherwise limit the efficiency at light loads. The matrix transformer resulted in highefficiencies without sacrificing the converter size and volume, as a high power density of $10 \mathrm{~kW} / 1$ (or $164 \mathrm{~W} / \mathrm{inch}^{3}$ ) together with a peak efficiency of $97.4 \%$ were ultimately achieved. (a)
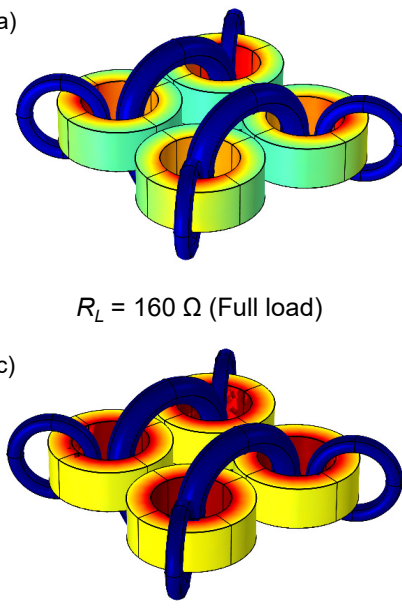

$R_{L}=1600 \Omega(10 \%$ of full load $)$

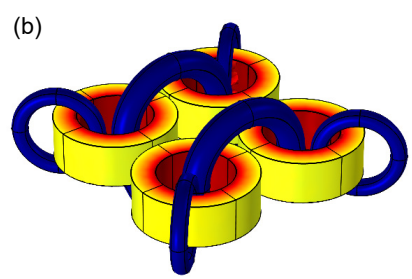

$R_{L}=800 \Omega(20 \%$ of full load $)$

(d)

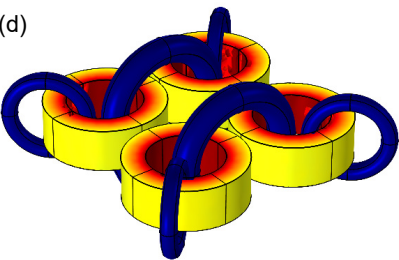

$R_{L}=3200 \Omega(5 \%$ of full load $)$

$$
\begin{array}{rllllllllll}
0 & 10 & 20 & 30 & 40 & 50 & 60 & 70 & 80 & 90 & 100 \\
& \multicolumn{1}{c}{B(\mathrm{mT})}
\end{array}
$$

Fig. 10 FEA results for magnitude and distribution of $B$ in the cores under different load conditions at a $40-\mathrm{V} 300-\mathrm{kHz}$ voltage excitation at the primary side of the transformer. (a) The full-load condition. After a certain increase in $R_{\mathrm{L}}, B$ remains almost similar for (b) $20 \%$, (c) $10 \%$ and (d) $5 \%$ of the full load. Thus, core losses do not increase drastically even at extremely light loads. 


\section{Operation of tap changers}

The switching transient of the employed EM relays is shown in Figs. 11a, b for operation under $28 \mathrm{~V}$ and $2.8 \mathrm{~A}$. As presented in Fig. 11a, it takes about $20 \mathrm{~ms}$ for the coil in the EM relay to be charged (the blue curve) and then the contact is activated (the red curve). Fig. 11b shows a contact switching time of $<5 \mathrm{~ns}$. In fact, the delay for the activation of the EM relays is much smaller than system-level dynamics in typical applications in which E-DAB converters operate, such as: photovoltaics (PV) energy harvesting with slow variations of temperature and solar irradiance over time, or Li-ion battery charging when a full charging cycle lasts for a few hours and battery voltage varies slowly over time.

The EM relays are also capable of on-load operation. For instance, Figs. 11c, e show the variation of the HV dc-link voltage $\left(v_{\mathrm{DC}}\right)$ for on-load opening and closing transients, respectively, and Figs. $11 \mathrm{~d}$, f provide a finer time scale for $v_{\mathrm{DC}}$ and $v_{\mathrm{DS}}$ (for a secondary-side transistor).
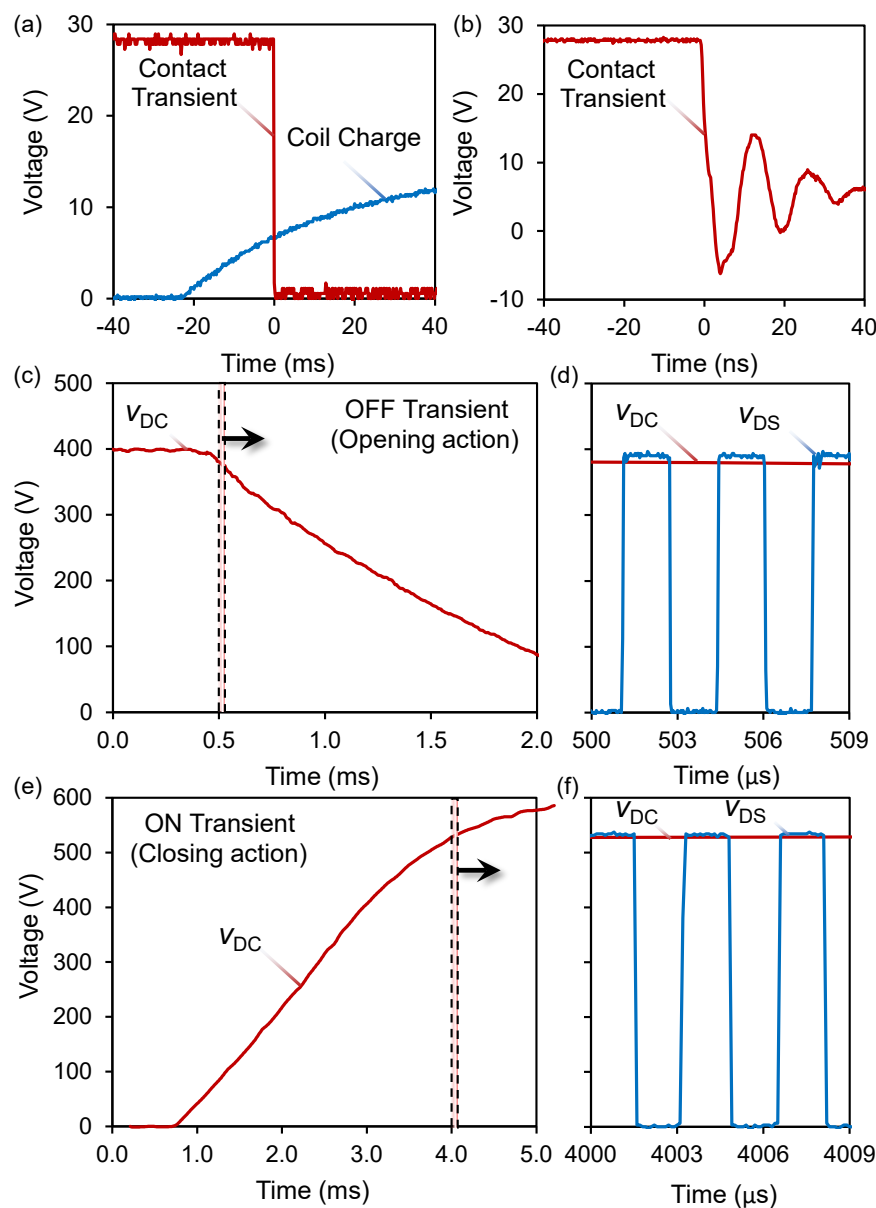

Fig. 11 Evaluation of the transient performance of the mechanical tap changer. (a) ms-scale demonstration of coil charging transient (blue curve) as well as contact voltage waveform (red curve). (b) ns-scale switching transient of the EM relay. (c), (d) Opening and (e), (f) closing transients of the output voltage in the E-DAB. Employed EM relays provide ns-range switching times after an activation delay of about $20 \mathrm{~ms}$; however, the dynamic behavior of the converter is constrained by the dc-link capacitance and load resistance. (c) and (e) show the HV dc-link voltage, where (d) and (f) provide the finer timescale for $v_{\mathrm{DC}}$ and $v_{\mathrm{DS}}$ of a secondary-side switching transistor.
The behavior of the converter is determined by the characteristics of the dc link, including its resistance (load) and capacitance.

The taps were placed at the HV side of the matrix transformer in order to minimize current stress on the EM relays. Off-load tap changing increases the lifetime of the EM relays. For better lifetimes and faster reaction times, one could employ solid-state switches in the tap-changer mechanism; however, the efficiency might be slightly lower [12].

\section{CONCLUSION}

We presented a full assessment of an enhanced DAB (EDAB) converter, which by utilizing an adjustable-tap highfrequency matrix transformer, increases power-transfer capability and extends soft-switching range over higher voltage gains. Two different transformer geometries were designed and compared for the amount and quality factor of their leakage inductances, where a matrix design with Litz wires exhibited a superior performance compared to a planar design with PCB traces. By employing the matrix transformer equipped with an electromagnetic tap changer, and by using the simplest modulation technique (single-phase-shift), the E-DAB converter achieved a peak efficiency of $97.4 \%$ with an overall efficiency always greater than that of a conventional DAB, for up to 2.8 times higher voltage gains under light-load operation. Experimental results were presented, where the analysis of switching transients and amplitude spectrum proved the effectiveness of using the turns ratio for minimizing the losses in the high-frequency E-DAB converter. The finite-element analysis of magnetic flux density in the ferrite cores were presented, where the proper sizing of the transformer resulted in low core losses and a high power density of $10 \mathrm{~kW} / \mathrm{l}$ (or 164 $\mathrm{W} /$ inch $^{3}$ ). We proposed a gain versus power-transfer characteristic plane which is of great importance to the design and control of E-DAB converters. We further demonstrated the compact design of GaN-based full bridges including the details on the PCB layout and driving stages. The transient behavior of the tap-changer mechanism was analyzed with two examples of on-load operation. The E-DAB topology provides superior performance in terms of flexibility, regulation capability and efficiency with applications in ultra-efficient Li-ion battery charging, renewable energy harvesting and $\mathrm{dc}$ voltage transformation in future dc distribution systems, among others.

\section{REFERENCES}

[1] L. Xue, Z. Shen, D. Boroyevich, P. Mattavelli, and D. Diaz, "Dual Active Bridge-Based Battery Charger for Plug-in Hybrid Electric Vehicle With Charging Current Containing Low Frequency Ripple," IEEE Trans. Power Electron., vol. 30, no. 12, pp. 7299-7307, Dec. 2015, doi: 10.1109/TPEL.2015.2413815.

[2] M. Forouzesh, Y. P. Siwakoti, S. A. Gorji, F. Blaabjerg, and B. Lehman, "Step-Up DC-DC Converters: A Comprehensive Review of Voltage-Boosting Techniques, Topologies, and Applications," IEEE Transactions on Power Electronics, vol. 32, no. 12, pp. 9143-9178, Dec. 2017, doi: 10.1109/TPEL.2017.2652318.

[3] A. Jafari, M. Samizadeh Nikoo, N. Perera, H. K. Yildirim, F. Karakaya, R. Soleimanzadeh, and E. Matioli, "Comparison of Wideband-gap Technologies for Soft-Switching Losses at High 
Frequencies," IEEE Trans. Power Electron., pp. 1-1, 2020, doi: 10.1109/TPEL.2020.2990628.

[4] M. Samizadeh Nikoo, A. Jafari, N. Perera, and E. Matioli, "Measurement of Large-Signal $C$ oss and $C$ oss Losses of Transistors Based on Nonlinear Resonance," IEEE Trans. Power Electron., vol. 35, no. 3, pp. 2242-2246, Mar. 2020, doi: 10.1109/TPEL.2019.2938922.

[5] A. Jafari and E. Matioli, "High Step-Up High-Frequency ZeroVoltage Switched GaN-Based Single-Stage Isolated DC-DC Converter for PV Integration and Future DC Grids," PCIM Europe 2018; International Exhibition and Conference for Power Electronics, Intelligent Motion, Renewable Energy and Energy Management, Nuremberg, Germany, 2018, pp. 1-6.

[6] A. Jafari, M. Samizadeh Nikoo, F. Karakaya, and E. Matioli, "Enhanced DAB for Efficiency Preservation Using Adjustable-Tap High-Frequency Transformer," IEEE Trans. Power Electron., vol. 35, no. 7, pp. 6673-6677, Jul. 2020, doi: 10.1109/TPEL.2019.2958632.

[7] N. Hou and Y. W. Li, "Overview and Comparison of Modulation and Control Strategies for a Nonresonant Single-Phase Dual-ActiveBridge DC-DC Converter," IEEE Trans. Power Electron., vol. 35, no. 3, pp. 3148-3172, Mar. 2020, doi: 10.1109/TPEL.2019.2927930.

[8] M. Yaqoob, K. H. Loo, and Y. M. Lai, "Extension of Soft-Switching Region of Dual-Active-Bridge Converter by a Tunable Resonant
Tank," IEEE Trans. Power Electron., vol. 32, no. 12, pp. 9093-9104, Dec. 2017, doi: 10.1109/TPEL.2017.2654505.

[9] Z. Qin, Y. Shen, P. C. Loh, H. Wang, and F. Blaabjerg, "A Dual Active Bridge Converter With an Extended High-Efficiency Range by DC Blocking Capacitor Voltage Control," IEEE Trans. Power Electron., vol. 33, no. 7, pp. 5949-5966, Jul. 2018, doi: 10.1109/TPEL.2017.2746518

[10] R. S. Yang, A. J. Hanson, B. A. Reese, C. R. Sullivan, and D. J. Perreault, "A Low-Loss Inductor Structure and Design Guidelines for High-Frequency Applications," IEEE Trans. Power Electron., vol. 34, no. 10, pp. 9993-10005, Oct. 2019, doi: 10.1109/TPEL.2019.2892397.

[11] A. Jafari, M. Samizadeh Nikoo, F. Karakaya, N. Perera, and E. Matioli, "97.4\%-Efficient All-GaN Dual-Active-Bridge Converter with High Step-up High-Frequency Matrix Transformer," PCIM Europe digital days 2020, International Exhibition and Conference for Power Electronics, Intelligent Motion, Renewable Energy and Energy Management, 2020, pp. 1-8.

[12] S. Taraborrelli, R. Spenke, and R. W. De Doncker, "Bidirectional dual active bridge converter using a tap changer for extended voltage ranges," in 2016 18th European Conference on Power Electronics and Applications (EPE'16 ECCE Europe), Karlsruhe, Sep. 2016, pp. 1-10, doi: 10.1109/EPE.2016.7695668. 